\title{
In Situ Exploration of Venus' Clouds by Dynamic Soaring
}

\section{Expanding Sampling Capabilities through Energy Harvesting}

Jack S. Elston ${ }^{1}$, Mark A. Bullock ${ }^{2}$, Maciej Z. Stachura ${ }^{3}$, Sebastien Lebonnois ${ }^{4}$, Sanjay S. Limaye ${ }^{5}$, David H. Grinspoon ${ }^{6}$, Michael Pauken ${ }^{7}$, Bruce Cogan ${ }^{8}$

Co-Signers:

Noam Izenberg ${ }^{9}$, Constantine Tsang ${ }^{10}$, Paul Byrne ${ }^{11}$, Amanda Brecht ${ }^{12}$, Jaime Cordova ${ }^{13}$, Ye Lu ${ }^{14}$, Jonathan Sauder ${ }^{15}$, Tibor Kremic ${ }^{16}$

\footnotetext{
${ }^{1}$ Black Swift Technologies, elstonj@bst.aero, 7206389656

${ }^{2}$ Science and Technology Corp, bullock@stcnet.com

${ }^{3}$ Black Swift Technologies

${ }^{4}$ Laboratoire de Météorologie Dynamique

${ }^{5}$ University of Wisconsin

${ }^{6}$ Planetary Science Institute

${ }^{7}$ Thermal and Fluid Systems, JPL

${ }^{8}$ NASA Armstrong Research Center

${ }^{9}$ Applied Physics Lab, Johns Hopkins University

${ }^{10}$ Southwest Research Institute

${ }^{11}$ NC State University

${ }^{12}$ NASA Ames Research Center

${ }^{13}$ UW Madison

${ }^{14}$ Kent State University

${ }^{15} \mathrm{JPL}$

${ }^{16}$ NASA GRC
} 


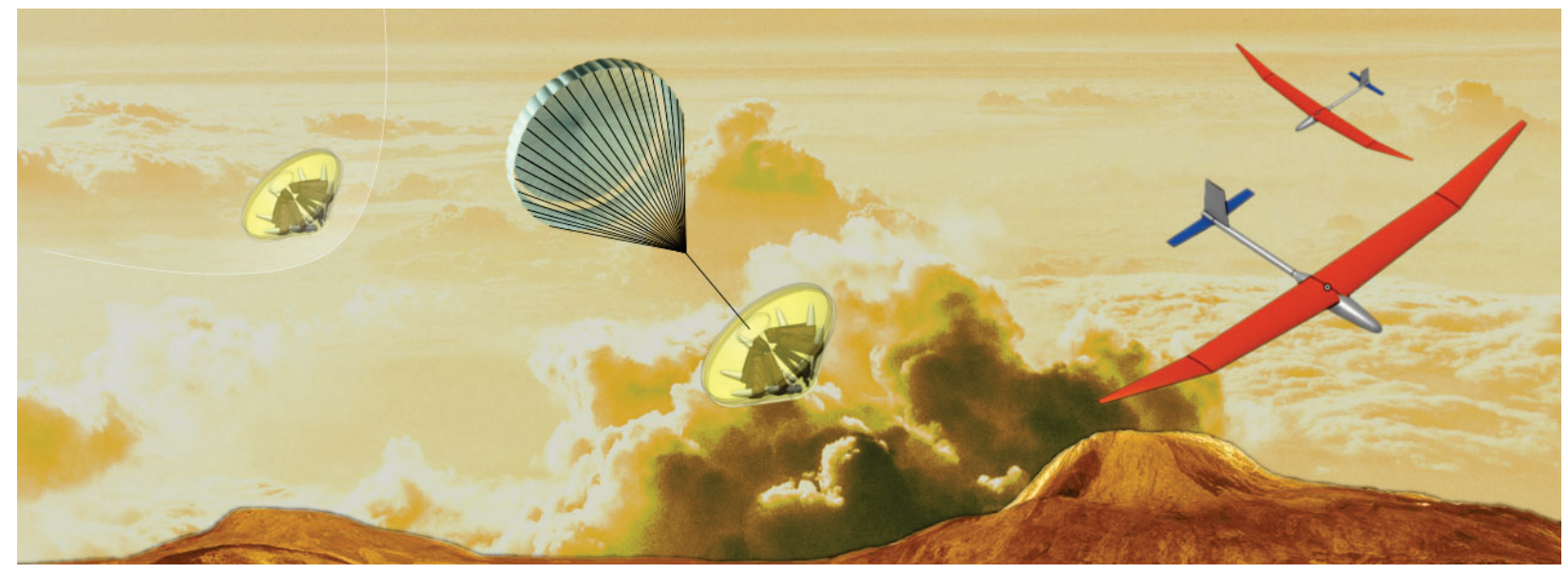

Figure 1: Deployment overview of a fixed-wing, energy harvesting vehicle, including atmospheric entry via aeroshell, parachute descent, and multiple air vehicle deployment.

Large gaps in our knowledge of the chemistry of Venus' clouds prevent an understanding of how the clouds affect its climate. The sulfur cycles below, within, and above the Venus clouds have been studied using PV GCMS data [1] and ever more sophisticated models [2,3]. Reconnaissance of Venus' clouds must involve a comprehensive analysis of the aerosols and sulfur chemistry in a wide range of locations within the atmosphere.

Models and direct observation of the atmosphere of Venus have shown that the environment above the cloud layer is incredibly dynamic [4-6]. The strong and persistent vertical shear conditions at Venus' cloud tops are ideal for sustained energy-harvesting soaring. Favorable environments for the use of soaring techniques provide energy to not only maintain altitude, but enable sufficient wind-relative velocity to navigate to desired global locations. Convective activity originating in the middle cloud is a persistent and global feature of Venus' atmosphere. [7-11] Additionally, large regions of Venus' atmosphere contain characteristically high vertical wind shear, particularly near the cloud tops [12]. This phenomenon can also be observed on Earth as it causes the capping of convection in convective storms [13] and the formation of high altitude cirrus clouds [14], and is evident above mountain waves [15].

Both updrafts and regions of high vertical shear can serve as sources of energy for a fixed wing aircraft. However, for a mission to range over a large area of the planetary surface, the use of gravity waves and equatorial updrafts has limited value to the sampling mission. Therefore, a current development program focuses on designing and testing aircraft and algorithms for harvesting energy near the Venus cloud tops. Intelligent flight path control can be used to combine dynamic soaring cycles in such a manner as to incrementally change position of the craft and provide nearly global mobility. Additionally, the flight path is ideal for systematic in situ study of Venus' clouds. On the upper branch of its trajectory, the aircraft traverses the upper cloud. The composition of gases and aerosols can be measured throughout this layer. The bottom of the arc is an ideal location for obtaining near-IR images of the surface and sampling the large particles in the lower cloud.

Of additional benefit is the simplification of operating on the dark side of the planet. Since propulsive power is supplied through the flight path, energy can be devoted to 
control surface actuation and on-board systems, generally a much smaller portion of the overall energy budget. It is plausible that making use of the abundant solar energy on the sunlit side could allow for charging batteries that last through the traversal of the night side. Furthermore, energy sources such as an external turbine will be considered that can provide energy regardless of the presence of sunlight. Finally, the use of rigid, deployable aircraft rather than inflatable structures simplifies the deployment mechanism. Rather than waiting for the vehicle to be inflated with a lighter-than-air gas, a simple mechanism can be used to deploy components from a compact configuration in a matter of seconds. This has been employed on Earth for tube launched UAS, which have been successfully deployed at very high relative speed over hurricanes for atmospheric research $[16,17]$.

\section{Science Case for In Situ Investigation of Venus' Clouds}

The clouds of Venus may be linked to past volcanism, and their stability over geological timescales is not well established. The clouds are a major reservoir of hydrogen and sulfur, but both these species are gradually lost from the atmosphere. Water vapor is lost at the top of the atmosphere, and $\mathrm{SO}_{2}$ reacts readily with atmospheric gases and with minerals at the surface.

Lee et al., 2019 [18] recently showed that the ultraviolet albedo of Venus varies on timescales of about 10 years. The unknown agent or agents responsible for these changes actually absorb over a broad spectral range and into visible wavelengths. The amplitude of these albedo changes was large - 40\% from 2006 to 2017 . These authors showed that the anomalous increase in zonal wind speeds that had been noted but not understood [19], can now be seen as due to increased absorption of sunlight in the clouds. This very large change in climate forcing over a decade was caused by the unknown near-UV absorber. The chemistry of Venus' atmosphere is therefore linked to the general circulation and to the distribution of its clouds.

VEXAG identified several high priority investigations that should be performed by an in situ mission to the clouds of Venus. The proposed vehicle would be ideal to address the second VEXAG goal to understand the dynamics and composition of Venus. Direct insertion and deployment into the winds at Venus' cloud tops would help to measure the dynamics of the deep atmosphere, accomplishing VEXAG Goals Objective, Investigation (GOI) II.A.DD. Additionally, flying in the clouds with sufficient mobility allows for investigating VEXAG GOI II.A.MP, mesoscale processes, and most of Goal II Objective B, to understand the atmospheric radiative balance, interactions between chemical species, cloud aerosols, the unknown absorber and volcanic gas input to the atmosphere. A vehicle capable of descending into the lower cloud deck could take near-IR images of the surface and obtain altimetry to produce images with 10 times the sharpness of current orbital images. Therefore, an in situ cloud mission can provide the necessary data to investigate the possible hydrous origins of surface minerals as well as support the search for geologic evidence of crustal recycling, VEXAG GOI I.A.HO and I.A.RE respectively. The proposed system will also be able to address VEXAG Goal III, to understand Venus geologic history and surface/atmosphere interactions. A vehicle capable of imaging the surface in near-IR from deep within the clouds (or below them) could provide new imaging and spectral data for achieving II.A, Understanding the geologic processes 
that shaped the surface of Venus, and II.B How do the surface and atmosphere interact?. Near-IR imaging coupled with an altimeter would provide higher fidelity mapping of the surface emissivity, and hence aid in VEXAG GOI III.A.GH, III.A.GC, and III.A.GA, and III.A.4. These are investigations of Venus' geologic history, geochemistry, and geologic activity. Investigations III.B.LW, local weathering, and III.B.GW, global weathering would also benefit from vastly improved near-IR imagery of the surface.

\section{Aerial Vehicles for Venus Exploration}

In situ investigations of Venus' atmosphere and clouds have been considered a high scientific priority for several decades [20]. A diverse array of vehicle types have been proposed [21]; a brief overview of those platforms is provided here.

Largely due to the success of the Soviet Vega balloons, a number of US researchers have proposed balloons with scientific payloads to fly in Venus' clouds [22]. However, balloons suffer from a number of disadvantages with respect to achieving the highest priority science at Venus. A survey of possible aerial platforms for Venus, the science that could be performed, and the technological challenges, was recently commissioned by NASA [21]. The in situ investigation of Venus' atmosphere and clouds can be facilitated in a number of ways scientifically, using fixed wing aircraft rather than balloons. The main differences, in order of priority, are: control of flight path for targeted investigations, greater controllable altitude range for in situ experiments, and unobstructed $4 \pi$ steradian field of view for optical investigations.

The extreme conditions of Venus' atmosphere have led some authors to propose vehicles that are hybrids of superpressure and zeropressure baloons [23]. A variety of phase change materials to adjust buoyancy over a wide range of pressures has also been suggested [21]. Variable altitude balloons are capable of exploring Venus' atmosphere from near the surface to the cloud tops, with some limited horizontal navigation. Although this provides greater ability to perform sampling across the desired altitude ranges, the transitions are relatively slow.

To the knowledge of this group, only one heavier than air vehicle for the exploration of Venus has been proposed [24-27]. This vehicle exclusively makes use of a large wing surface area for providing solar power to an electric propulsion system. To solve the issue of not being able to operate for extended periods of time on the night side of the planet, the authors proposed that it could be possible to fly faster than the wind speeds around the desired sampling altitudes between 40 and $60 \mathrm{~km}$. While this may be possible to achieve, it does limit the sampling range of the aircraft, and relies heavily on the fact that the efficiency of the solar power system will not degrade during long flights through the atmosphere.

\section{Persistent Exploration with Dynamic Soaring}

Autonomous soaring has been shown in both simulation and flight tests to increase the endurance of small UAS. Recent work has focused on the development of automatic control algorithms for harvesting energy from relatively static features such as thermals [28] or ridge lift [29]. These algorithms exploit the structure of updrafts to keep the 
aircraft within an area where it can gain energy, leaving the updraft to perform any secondary tasks, or when the updraft's potential has dissipated. Other efforts have focused on utilizing wind-field gradients to add energy to the aircraft [30-32]. That work generally considers short flight paths designed to benefit from the structure of the wind field, and links the flight paths to a set of sensing goals. Limited work has also focused on energy extraction from gusts [33]. This requires a reactive technique to make use of the short term effects of turbulence to impart small amounts of energy to the vehicle.

Dynamic soaring is achieved by climbing into an increasing wind gradient and turning back to descend through it. Given a large enough gradient and appropriate control of the flight trajectory this method can be used to harvest energy from the atmosphere without the use of on-board propulsion. This makes the practice particularly interesting for application to the exploration of Venus' atmosphere, where strong shear layers occur around the desired altitudes and span a large portion of the planet.

A simulation environment was created using a candidate aircraft model and control algorithms to achieve trajectories required for dynamic soaring through tracking an inclined orbit. The controller allowed variations of the tilt angle of the orbit, the radius, and the speed of the aircraft. Although wind speed from Venus models show that aircraft of the desired scale will not be capable of station keeping, the concept of flying an inclined orbit is still possible when considered in the wind frame.

For these simulations the aircraft airspeed was set to be constant and the excess energy generated was logged. In reality this energy would only be of benefit if it was captured by the system, through the acceleration of the aircraft, or converted to electricity by an on-board turbine. However, the purpose of these simulations was to compare trajectories and demonstrate energy gain. For that reason a total instantaneous power is calculated based on what is needed to maintain airspeed. It is then integrated and combined with kinetic and potential energy of the aircraft to assess how much energy the aircraft is gaining or losing along the trajectory.

The simulated flights made use of several different sources for generation of atmospheric parameters. The most simple representation consisted of wind fields that were based on probe data, essentially modeling the wind regime at the cloud tops. More detailed atmospheric models were also considered such as the one from Baker [7], which adds a convection component.

Of greater interest is the use of Large Eddy Simulations (LES) [lef] to generate atmospheric parameters. This model contains a significant amount of detail, based mainly on the results of modeling the convective plumes $(3 \mathrm{~m} / \mathrm{s})$ measured by the Vega balloons. These plumes occur mostly in the $46-55 \mathrm{~km}$ altitude range, where most of our flights are to occur. The LES provides a more accurate representation of the anticipated atmospheric conditions. Mesoscale models [34] and general circulation models (GCM) [35] can be employed to support planet-wide mission analysis. The flexibility of the flight simulation software also allows for the use of data from other GCMs, and the ingestion of data from empirical models such as Venus-GRAM, to be incorporated in future work.

One of the largest risks for the system is the assumption that sufficient shear exists to maintain flight for the duration of the mission. Based on probe data and the latest GCM results, this seems to be a reasonable assumption, but further investigation is necessary. Alternative emergency propulsion methods could be considered, such as through 

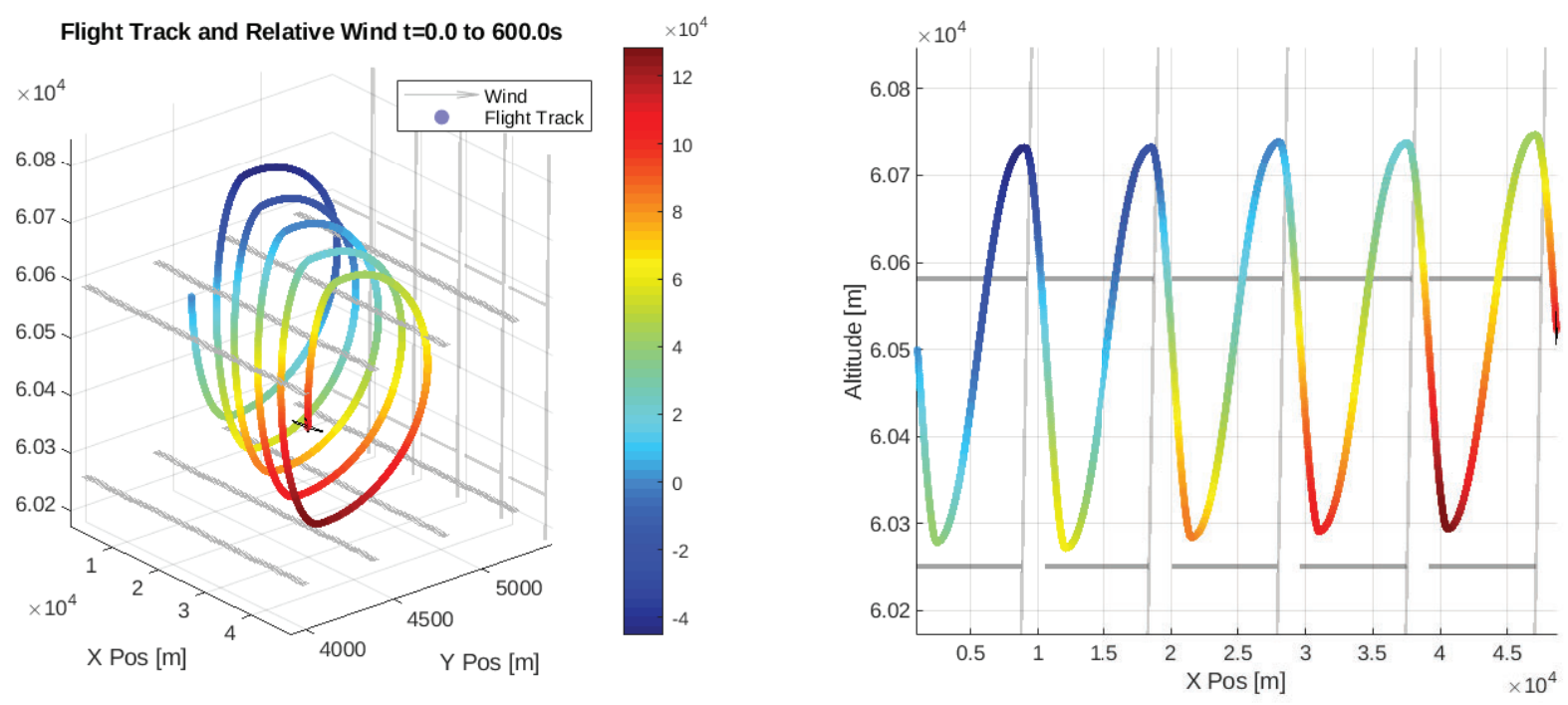

Figure 2: Results from a flight through the high fidelity model using the relative version of the dynamic soaring algorithm. The flight was conducted around $60 \mathrm{~km}$ in altitude over 600 seconds and shows relative energy gain as the color of the flight path.

a radioisotope thermoelectric generator (RTG) or on-board battery that is charged using either a RTG, solar panels on the wings, or through a turbine that can be introduced into the stream during periods of strong energy gain by the aircraft.

Another risk is determining the location of the aircraft relative to the atmospheric structures it is using for energy harvesting. The possibility of including a small orbiter at one of the Lagrange points for localizing the aircraft has been considered. Additionally, techniques are being tested to determine the wind field simply through analysis of onboard sensors.

Lastly, the aircraft will have to survive the Venus environment. Although the intended sampling mission would be performed in a regime that remains within operational pressure and temperature limits for materials and mechanisms commonly used on Earth, the corrosive environment presents a significant challenge. Work has begun to investigate survivability of various composites in this environment. Thin coatings for protection that could allow for more conventional building materials are being investigated.

The aforementioned dynamic soaring algorithm was used to conduct several simulated flights through data generated from the latest Weather Research and Forecasting (WRF) large eddy simulation that is part of the above GCM. Two sets of simulations were run, one at $55 \mathrm{~km}$ altitude and the other at around $60 \mathrm{~km}$. $55 \mathrm{~km}$ was chosen as it is situated in the middle of the regime identified as scientifically significant. $60 \mathrm{~km}$ was chosen due to the large amount of vertical shear in the region. The plots in Figure 2 show the results from one $60 \mathrm{~km}$ flight.

Flights at both altitudes demonstrated a net energy gain. What is interesting to note about the results is that the average energy gathered per cycle was larger at the $60 \mathrm{~km}$ altitude, but the total energy gain in the system was larger for the flight at $55 \mathrm{~km}$. Upon further examination, it became apparent that this was due to the presence of a positive 
vertical wind component through this part of the simulation.

Additionally, the simulated data were used to determine if anticipated downdrafts would result in the inability of the aircraft to maintain altitude to within a reasonable level. Examining the results from the GCM at $59 \mathrm{~km}$ reveals that generally the magnitude of the vertical winds was around $3 \mathrm{~m} / \mathrm{s}$, making them small enough to have only a minor effect on total system energy.

Therefore, although greater understanding of the Venus atmosphere would improve the ability to predict the performance of the proposed craft, current data suggests it should be possible to achieve sustained flight as well as the ability to perform targeted sampling.

\section{Payload and Mission Design}

One of the main challenges for the mission is the design of the aircraft to fit within the vehicle designed to carry it into the upper atmosphere of Venus. It is assumed at this point that deployment will occur after slowing to sub-sonic speeds through aerobreaking and possibly the use of a parachute. Because of this assumption, the initial design for the vehicles has been for installation into a aeroshell. A candidate deployment setup has been designed to fit in the aeroshell used by Pioneer Venus. This configuration accomodates several aircraft assuming that a mechanism can be found that will allow for the high aspect ratio wings to be folded and then deployed upon release. Risk factors exist for this design as the aircraft will have to deal with relatively high g-loading during flight, and survive the corrosive environment.

Another challenge is wind field estimation for use in generation of energy harvesting flight paths. This will be made possible by combining vehicle in situ measurements with absolute location determined by tracking from Earth-based radio dishes and from spacecraft in the vicinity of Venus. Lagrange point orbiters, for example, would be ideal for relaying high bandwidth data from aircraft in the clouds.

The scientific demands of an in situ mission to Venus' clouds are broad. Payload mass is limited for any aerial platform. Extremely small $(10 \mathrm{~g})$ chemical sensors have been developed at Glenn Research Center, and miniaturized mass spectrometers have been developed at JPL that could fly on such a vehicle. Gas and aerosol handling in a highly corrosive environment is a crucial part of any in situ mission to the clouds of Venus, and will have to be developed for any future mission. In order to address the VEXAG Goals, Objective, and Investigations discussed in Section 2, optical instrument to measure sky flux, count aerosols, and determine their indices of refraction will also be needed.

\section{Conclusions}

The deeper mysteries of Venus' climate can only be solved by performing in situ investigations in Venus' clouds. Balloons, descent probes, fixed-wing aircraft, aerobots, and rotorcraft have all been proposed to achieve some of the VEXAG scientific objectives. To date, only the 2 Vega balloons and 16 entry probes have returned data from Venus. The chances for scientific success increase if the aerial vehicle has longevity, can fly at night, and is capable of navigating to interesting regions of the Venus atmosphere. 
Acknowledgements: This research was supported by NASA Phase II SBIR grant 80NSSC19C0181 to Black Swift Technologies, and NASA grant NNX16AC81G to Bullock.

\section{References}

[1] Oyama, V. et al. Journal of Geophysical Research: Space Physics 85, 7891-7902 (1980). [2] Mills, F. P. et al. (2007). [3] Krasnopolsky, V. A. Icarus 299, 294-299 (2018). [4] McGouldrick, K. et al. Icarus 286, 118-133 (2017). [5] Tellmann, S. et al. Journal of Geophysical Research: Planets 114 (2009). [6] Sánchez-Lavega, A. et al. Geophysical Research Letters 35 (2008). [7] Baker, R. D. et al. Journal of the atmospheric sciences 55, 3-18 (1998). [8] Ainsworth, J. et al. Journal of Geophysical Research 80, 173-179 (1975). [9] Schubert, G. et al. Journal of Geophysical Research: Space Physics 85, 80078025 (1980). [10] Fedorova, A. et al. Icarus 275, 143-162 (2016). [11] Lefèvre, M. et al. Journal of Geophysical Research: Planets 123, 2773-2789 (2018). [12] Hueso, R. et al. Icarus 217, 585-598 (2012). [13] Marwitz, J. D. Journal of Applied Meteorology 11, 166-179 (1971). [14] Jensen, E. J. et al. Journal of Geophysical Research: Atmospheres 101, 21361-21375 (1996). [15] Eckermann, S. D. et al. Quarterly Journal of the Royal Meteorological Society: A journal of the atmospheric sciences, applied meteorology and physical oceanography 133, 1959-1975 (2007). [16] Cione, J. et al. Earth and Space Science 3, 370-380 (2016). [17] Bryan, G. et al. Unique Observations in Hurricane Maria (2017) using the Coyote Uncrewed Aircraft System (UAS) in AGU Fall Meeting Abstracts (2017). [18] Lee, Y. J. et al. The Astronomical Journal 158, 126 (2019). [19] Peralta, J. et al. The Astrophysical Journal Supplement Series 239, 29 (2018). [20] Herrick, R. et al. VEXAG, Goals, Objective, and Investigations for Venus Exploration tech. rep. (NASA, 2014). [21] Cutts, J. A. et al. Aerial Platforms for the Scientific Exploration of Venus tech. rep. (Venus Aerial Platforms Study Team, 2018). [22] Baines, K. H. et al. (2005). [23] Rabinovitch, J. et al. Aerobots to Explore Venusian Clouds in Venera-D Landing Sites Selection and Cloud Layer Habitabiliy Workshop (Moscow, Russia, 2019). [24] Landis, G. A. Exploring Venus by solar airplane in AIP Conference Proceedings 552 (2001), 1618. [25] Landis, G. et al. Atmospheric flight on Venus in 40th AIAA Aerospace Sciences Meeting \& Exhibit (2002), 819. [26] Landis, G. A. et al. Journal of Spacecraft and Rockets 40, 672-677 (2003). [27] Ashish, K. et al. Flight analysis of a Venus atmospheric mobile platform in Aerospace Conference, 2014 IEEE (2014), 1-14. [28] Andersson, K. et al. Cooperating UAVs Using Thermal Lift to Extend Endurance in AIAA Infotech@Aerospace Conference (Seattle, WA, Apr. 2009). [29] Sachs, G. et al. Optimization of dynamic soaring at ridges in AIAA Atmospheric Flight Mechanics Conference and Exhibit, Austin, Texas (2003), 11-14. [30] Sachs, G. Ibis 147, 1-10 (2005). [31] Lissaman, P. AIAA paper 241 (2005). [32] Richardson, P. L. Progress in Oceanography 88, 46-58 (2011). [33] Langelaan, J. W. et al. Journal of Guidance, Control, and Dynamics 34, 1016-1030 (2011). [34] Lefèvre, M. et al. Icarus 335, 113376 (2020). [35] Lebonnois, S. et al. Journal of Geophysical Research: Planets 115 (2010). 